PS $\quad$ PROCEEDINGS

\title{
SPIDER: A Balloon-Borne Polarimeter for Measuring Large Angular Scale CMB B-modes
}

Carrie MacTavish*, Dick Bond, Olivier Doré

CITA, University of Toronto, Canada

E-mail: cmactavi@cita.utoronto.ca

Rick Bihary, Tom Montroy, John Ruhl

Department of Physics, Case Western Reserve University, USA

Peter Ade, Carole Tucker

Cardiff University, U.K.

James Bock, Warren Holmes, Jerry Mulder, Anthony Turner

NASA, Jet Propulsion Laboratory, USA

Justus Brevik, Abigail Crites, Sunil Golwala, Viktor Hristov, Bill Jones, Chao-Lin Kuo, Andrew Lange, Peter Mason, Amy Trangsrud

Division of Physics, Math and Astronomy, California Institute of Technology, USA

\section{Carlo Contaldi}

Theoretical Physics Group, Imperial College, UK

\section{Brendan Crill}

IPAC, California Institute of Technology, USA

\section{Lionel Duband}

Commissariat A L'energie Atomique, France

Mark Halpern

Deptarment of Physics and Astronomy, University of British Columbia, Canada 


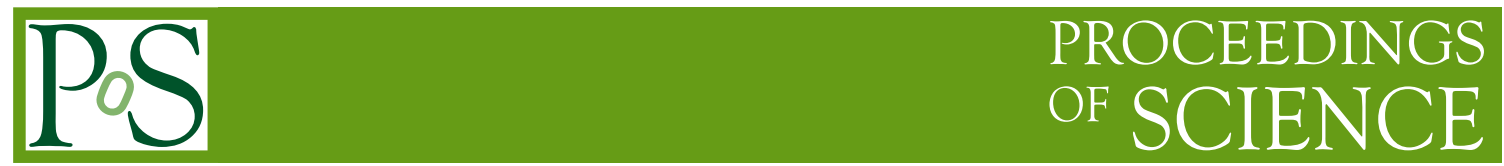

\section{Gene Hilton, Kent Irwin}

National Institute of Standards and Technology, USA

\section{Barth Netterfield, Enzo Pascale, Marco Viero}

Department of Physics/Astronomy and Astrophysics, University of Toronto, Canada

SPIDER is a balloon-borne polarimeter that is designed to measure CMB B-mode polarization. The experiment is scheduled to launch from Alice Springs, Australia in December of 2009 and will target large angular scales where the B-mode signal from primordial gravity waves dominates. In a mid-latitude, around the world, 25 day flight SPIDER will map out roughly $50 \%$ of the sky. The instrument will observe in five freqencies ranging from $80 \mathrm{GHz}$ up to $275 \mathrm{GHz}$ enabling accurate characterization of the interstellar dust foreground.

$C M B$ and Physics of the Early Universe

20-22 April 2006

Ischia, Italy

${ }^{*}$ Speaker. 


\section{Introduction}

SPIDER is a balloon-borne millimeter-wave polarimeter that targets the large angular scale B-mode polarization of the CMB. Probing CMB B-modes effectively probes the energy scale of Inflation, addressing the most fundamental of questions. SPIDER will also measure the curl-free E-mode component of the CMB. Constraining the amplitude of the low- $\ell$ Reionization peak of the EE angular power spectrum will significantly improve the determination of the Compton depth, $\tau$, and possibly th details of the Reionization epoch. As a multi-frequency experiment SPIDER will measure the polarized emission from interstellar dust with unprecedented precision. This represents a crucial step toward the accurate characterization of CMB foregrounds essential to any space mission that would target the tiny CMB polarization signal.

\section{The Spider Instrument and Flight}

The instrument, depicted in Figure 1, consists of 6 monochromatic telescope inserts enclosed in a single LN/LHe cryostat. SPIDER will spin in azimuth with telescopes fixed in elevation. The experiment builds on the legacy of several successful suborbital experiments. For example the payload attitude control system will use the same basic designs incorporated in the BLAST and BOOMERANG experiments. The SPIDER optical train, show in Figure 2 is based on the wellcharacterized BICEP design. A rotating half-wave plate, placed at each telescope aperture, will provide ideal polarization modulation. For detectors SPIDER will use a new generation of antennacoupled bolometer arrays. The SPIDER flight plan is to launch from Alice Springs, Australia in December 2009, maintaining constant latitude, traveling around the world in roughly 25 days.

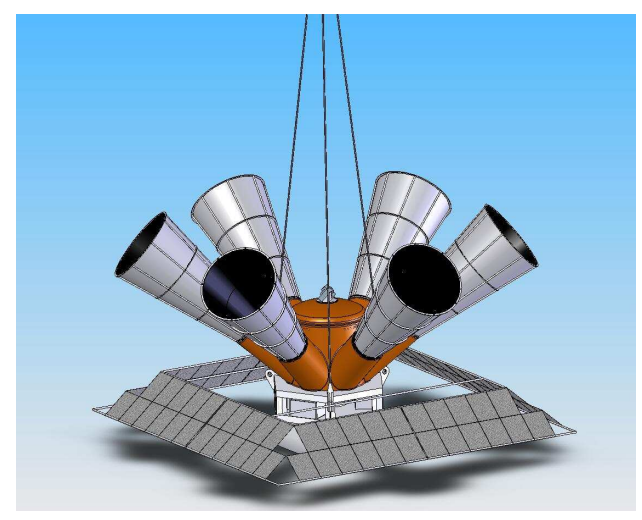

Figure 1: The SPIDER payload is designed to obtain maximum sky coverage from a twenty to thirty day mid-latitude balloon flight around the world. The experiment will map out roughly $50 \%$ of the sky observing only at night.

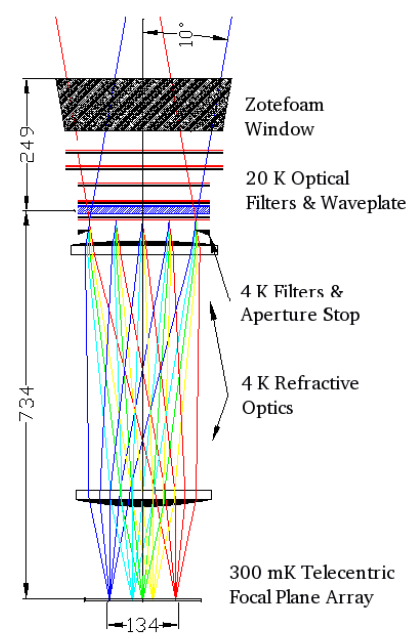

Figure 2: The SPIDER optics are based on the BICEP design. SPIDER will incorporate a rotating half-wave plate to modulate incoming polarization signal. 


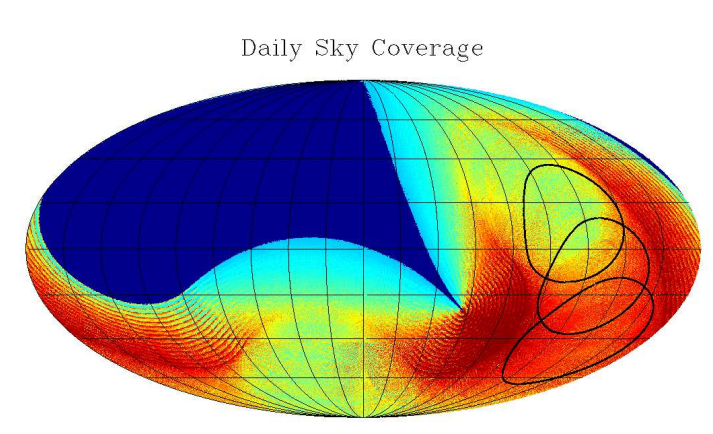

Figure 3: Simulated SPIDER sky coverage for a mid-latitude, 25 day flght, observing only at night.

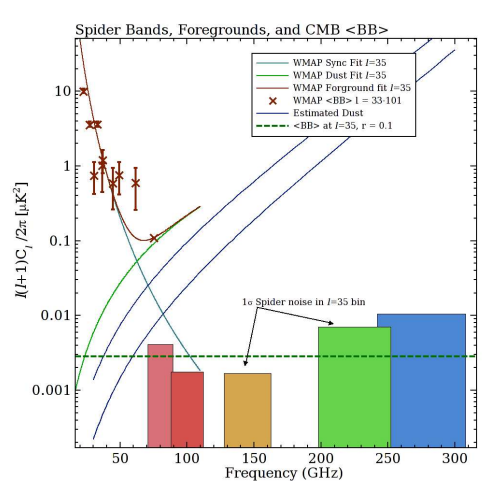

Figure 4: SPIDER $1 \sigma$ sensitivities in each of the 5 observing frequencies at $\ell=35$. Curves are estimates for foreground dust (rising with frequency) and synchrotron (falling with frequency). The data points are from the WMAP 3 year data [1].

SPIDER aims to map out approximately $50 \%$ of the sky. Figure 3 shows the simulated SPIDER sky coverage for 16 detectors spread over the bolometer array's range in elevation.

\section{Astrophysical Foregrounds}

Astrophysical foregrounds will be the biggest challenge to obtaining high precision measurements of CMB polarization. At high frequencies the polarized emission from dust dominates and at low frequencies polarized synchrotron emission dominates. SPIDER meets this challenge by observing, with high sensitivity, in 5 bands ranging in frequency from $80 \mathrm{GHz}$ up to $275 \mathrm{GHz}$. Figure 3 summarizes the current knowledge of polarized foreground emission. The vertical bars show SPIDER $1 \sigma$ sensitivities at $\ell=35$ at each of the observing frequencies. A summary of SPIDER observing bands, including beam sizes, bandwidths, number of pixels/detectors in each band and detector/instrument sensitivities is given in Table 1.

\section{SPIDER Science}

SPIDER's primary scientific goal is to measure, with high fidelity, the CMB polarization on

\begin{tabular}{|c|c|c|c|c|c|c|}
\hline $\begin{array}{c}\text { Observing } \\
\text { Band } \\
(\mathrm{GHz})\end{array}$ & $\begin{array}{c}\text { Bandwidth } \\
(\mathrm{GHz})\end{array}$ & $\begin{array}{c}\text { Beam } \\
\text { FWHM } \\
(\operatorname{arcmin})\end{array}$ & $\begin{array}{c}\text { Number of } \\
\text { Spatial } \\
\text { Pixels }\end{array}$ & $\begin{array}{c}\text { Number of } \\
\text { Detectors }\end{array}$ & $\begin{array}{c}\text { Single-Detector } \\
\text { Sensitivity } \\
\left(\mu \mathrm{K}_{\mathrm{CMB}} \sqrt{\mathrm{s}}\right)\end{array}$ & $\begin{array}{c}\text { Instrument } \\
\text { Sensitivity } \\
\left(\mu \mathrm{K}_{\mathrm{CMB}} \sqrt{\mathrm{s}}\right)\end{array}$ \\
\hline \hline 80 & 19 & 72 & 100 & 200 & 110 & 7.8 \\
100 & 24 & 58 & $(2 \times) 144$ & $(2 \times) 288$ & 100 & 4.2 \\
145 & 35 & 40 & 256 & 512 & 100 & 4.4 \\
225 & 54 & 26 & 256 & 512 & 204 & 9.0 \\
275 & 66 & 21 & 256 & 512 & 351 & 15.5 \\
\hline \hline
\end{tabular}

Table 1: Summary of SPIDER observing bands. Instrument sensitivities are derived by dividing the single detector sensitivity by the square root of the number of detectors. SPIDER will have a total of 2312 detectors observing at 5 different frequencies. 

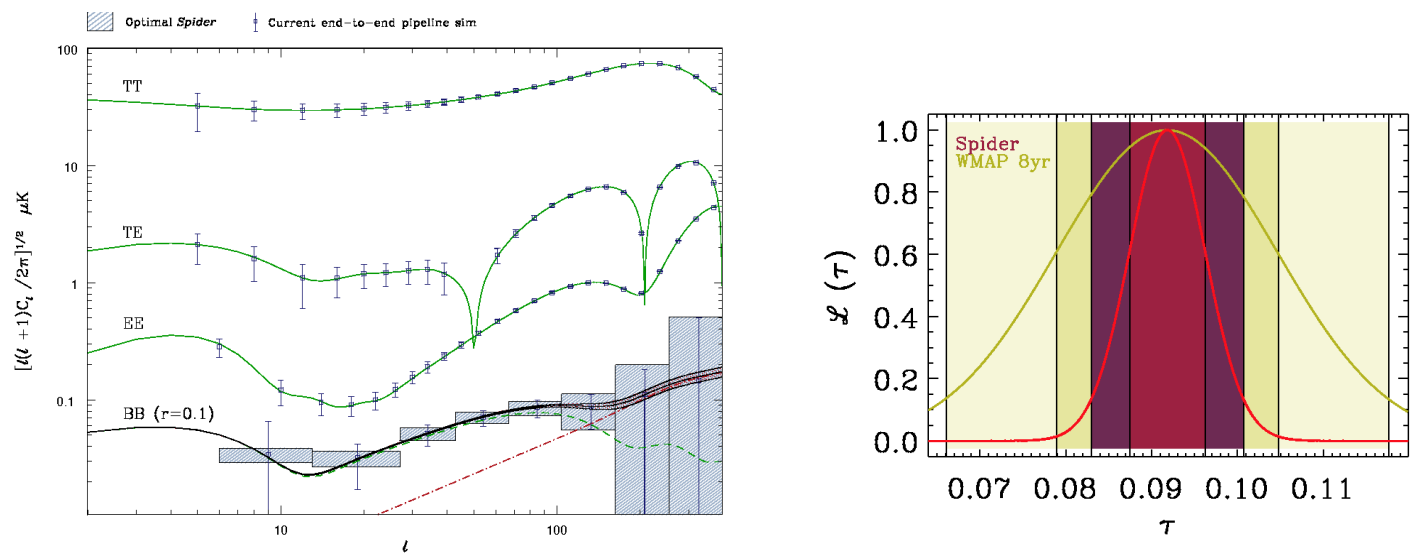

Figure 5: Top left panel: SPIDER sensitivity. Data points and errors are derived from end-to-end realizations of a simulated SPIDER balloon flight. Minimal modifications to the existing pipeline will result in an improved accuracy of the low multipole BB: the errors will approach the analytical errors (blue boxes) which are fully optimal with respect to the leakage of EE into BB. Top right panel: One-dimensional constraints on $\tau$. Bottom right panel: Two dimensional constraints

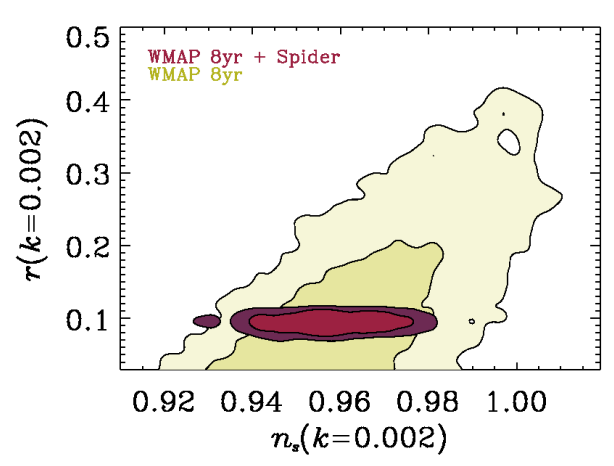
in the $n_{s}-r$ plane.

large angular scales. By aiming for large scale B-modes SPIDER minimizes the contamination to the BB angular power spectrum from B-mode induced by gravitational lensing. Figure 5 (left panel) illustrates the accuracy with which SPIDER will measure the TT, TE, EE and BB angular power spectra. We consider the effects on cosmic parameters in a minimal inflation model, with a uniform power law primordial index $\left(n_{s}\right)$ and a single tensor-to-scalar ratio, $r$ (it may well be that the inflation model is more complex in which case the high precision determination from SPIDER will be even more essential for unraveling the gravity wave induced contribution). From the EE spectrum alone SPIDER will improve the WMAP 8 year constraint on $\tau$ by more than a factor of three (figure 5 top right panel). The SPIDER constraints on $r$ will break one of the more sever degeneracies (figure 5 bottom right panel) remaining in CMB data in the $n_{s}-r$ plane. SPIDER constraints on $\tau$ and $r$ represent a reduction in the allowed volume in the $n_{s}-\tau-r$ parameter space by a factor greater then 50 .

\section{References}

[1] Page, L. et al., "Three Year Wilkinson Microwave Anisotropy Probe (WMAP) Observations: Polarization Analysis", submitted to Astroph. J., astro-ph/0603450 